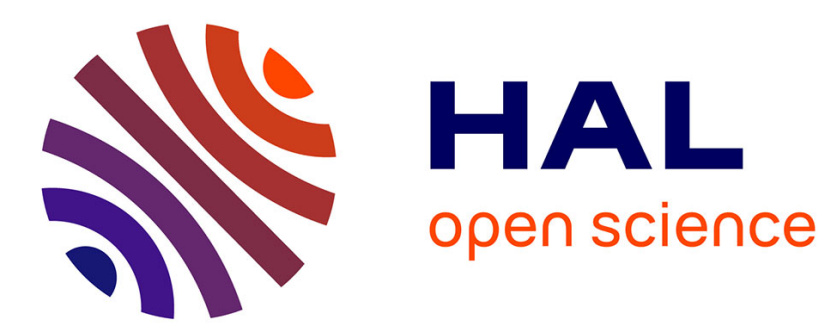

\title{
INHARMONICITÉ ET SAXOPHONE QUART DE TON
}

Joel Gilbert, Jean-Pierre Dalmont

\section{To cite this version:}

Joel Gilbert, Jean-Pierre Dalmont. INHARMONICITÉ ET SAXOPHONE QUART DE TON. Journal de Physique IV Proceedings, 1992, 02 (C1), pp.C1-71-C1-74. 10.1051/jp4:1992111 . jpa-00251064

\section{HAL Id: jpa-00251064 https://hal.science/jpa-00251064}

Submitted on 1 Jan 1992

HAL is a multi-disciplinary open access archive for the deposit and dissemination of scientific research documents, whether they are published or not. The documents may come from teaching and research institutions in France or abroad, or from public or private research centers.
L'archive ouverte pluridisciplinaire HAL, est destinée au dépôt et à la diffusion de documents scientifiques de niveau recherche, publiés ou non, émanant des établissements d'enseignement et de recherche français ou étrangers, des laboratoires publics ou privés. 


\section{INHARMONICITE ET SAXOPHONE QUART DE TON}

\section{J. GILBERT et J.P. DALMONT}

Laboratoire d'Acoustique de l'Université du Maine, CNRS URA 1101, U.F.R. Sciences, BP. 535, Université du Maine, Avenue Olivier Messiaen, F-72017 Le Mans cedex, France

Résumé: En mode de jeu normal, la fréquence de jeu d'un instrument à anche est proche d'une des fréquences de résonance du résonateur. La facilité d'émission est reliée à l'harmonicité des premières fréquences de résonance. Nous montrons dans les deux cas qu'il est plus satisfaisant de considérer le résonateur avec un volume équivalent à l'anche à l'entrée plutôt que le résonateur seulement.

Abstract: The playing frequency of a reed instrument, in normal playing mode, is near the resonance frequency of the resonator. The ease of playing is related to the harmonicity of the first resonance frequencies. We show here that for both case it is more satisfying to consider the resonator added with the equivalent volume to the reed instead of the resonator only.

\section{1-Introduction}

L'instrument à vent à anche simple (clarinette, saxophone) peut être décrit comme un système physique autooscillant. Il est essentiellement constitué d'un résonateur (le corps de l'instrument) couplé à un système excitateur (l'ouverture anche-table du bec). En mode de jeu normal, la fréquence de jeu du système est voisine d'un des maximums de la courbe $d$ 'impédance $d$ 'entrée $Z$ e du résonateur. Bouasse $/ 1 /$ puis Benade $/ 2 /$ ont noté que la facilité d'émission est liée à une bonne harmonicité des fréquences de résonance du résonateur. Nous montrons ici que cette remarque est juste mais en considérant les résonances du résonateur en parallèle avec l'impédance d'anche plutôt que celle du résonateur seule.

\section{2- Impédance d'anche}

En mode de jeu normal la fréquence de jeu est inférieure à la fréquence de résonance correspondante. On peut vérifier expérimentalement $/ 3 /$ que le terme de débit d'anche, débit de moyenne nul provoqué par le mouvement de l'anche à l'intérieur du bec, est la cause essentielle du décalage fréquentiel. On peut traduire l'effet du débit d'anche par la prise en compte d'une impédance $Z a$ appelée impédance d'anche, en parallèle avec l'impédance $d$ 'entrée $Z_{e}$ du résonateur. A basse fréquence, $Z a$ est équivalente à un volume supplémentaire $V$ à l'entrée de l'instrument $/ 2 /$ ou à une correction de longueur $\Delta \ell$ telle que $V=S \Delta \ell / 4 /$ (S est la section d'entrée du résonateur). 
Au seuil d'oscillation on montre que l'écart fréquentiel relatif entre fréquence de jeu et fréquence de résonance du résonateur $\Delta F / F$ est :

$$
\frac{\Delta F}{F}=-\frac{2 F}{\left(n-\frac{1}{2}\right)} \frac{\Delta \ell}{c}
$$

où c est la vitesse du son; n est l'ordre de la fréquence de résonance $F$. Il est possible d'extrapoler la relation (1) au delá du seuil, pour une embouchure fixée, avec une sérle de résonateurs cylindriques de longueur variable (figure 1 ).

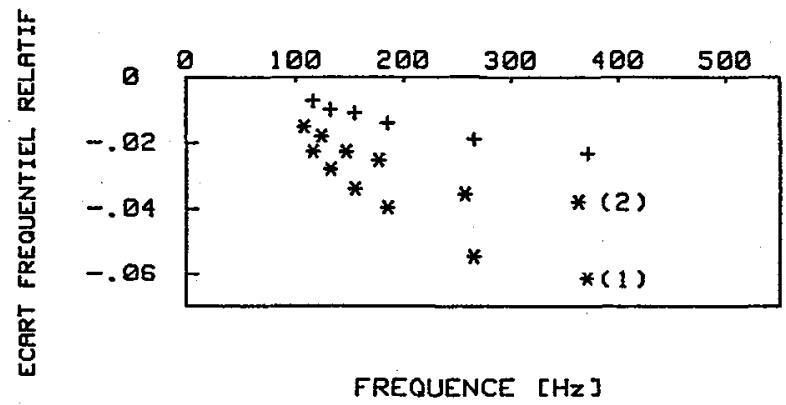

Figure 1 : Ecart fréquentiel relatif $\triangle F / F$ entre la fréquence de jeu du système et la fréquence de résonance du résonateur pour un jeu de tubes cylindriques de longueur variable. Résultats expérimentaux (*) pour deux embouchures : une embouchure "relachée" (1) et une embouchure "pincée" (2), les résultats expérimentaux différence des deux embouchures sont représentés par $(+)$.

Nous avons montré expérimentalement qu'il est possible de caractériser une embouchure, en première approximation, par une correction de longueur $\Delta \ell$ indépendante du résonateur. On constate qu' une embouchure "relachée" implique une correction de longueur $\Delta l$ plus importante qu'une embouchure "pincée".

3- Impédance d'entrée à deux pics d'impédance : influence de 1'harmonicité des résonances sur la facilité d'émission

3-1 Le doigté de sol grave du saxophone alto

Nous présentons figure 2 la courbe d'impédance d'entrée mesurée du sol grave du saxophone alto. 


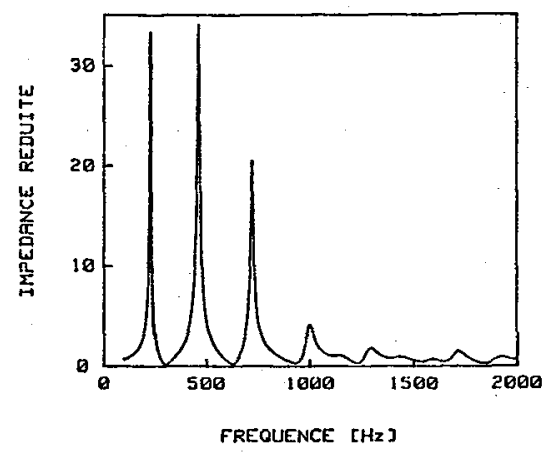

Figure 2 : Courbe expérimentale de l'impédance d'entrée réduite du doigté de sol grave du saxophone alto de 50 à $2000 \mathrm{~Hz}$. Le bec est remplacé par un tube cylindrique de diamètre $16 \mathrm{~mm}$ et de longueur $40 \mathrm{~mm}$. $F_{1}=230.5 \mathrm{~Hz}$ avec $Z=33.4$ (première résonance) et $F_{2}=463.9 \mathrm{~Hz}$ avec $Z=34.1$ (deuxième résonance).

3-2 Rôle de l'harmonicité des deux fréquences de résonance sur

\section{l'oscillation}

Lors de la mise au point du saxophone quart de ton, nous avons été amené à décaler la première fréquence de résonance $F$ du doigté de sol grave d'un quart de ton. Pour cela nous avons disposé ${ }^{1}$ deux tubes fermés de hauteur variable, en dérivation sur le bocal du saxophone : un premier tube (tube A) de diamètre $12 \mathrm{~mm}$ placé à $40 \mathrm{~mm}$ de l'entrée du bocal, un second tube (tube $B$ ) de díamètre $12 \mathrm{~mm}$ placé à $165 \mathrm{~mm}$ de l'entrée du bocal.

La mise en oeuvre progressive (hauteur $h$ croissante) du système $B$ provoque un abaissement de la fréquence de jeu accompagnée d'une détérioration du timbre du sol grave. Lorsque $\mathrm{h}=65 \mathrm{~mm}$ (hauteur prévue pour décaler $F$ d'un quart de ton), il $y$ a bifurcation d'un régime périodique vers un régime quasi-périodique ("note qui roule") cas limite d'une note difficile à émettre. Par contre la mise en oeuvre progressive du système A conserve à la fois le timbre et la facilité d'émission du sol grave, tout en provoquant une translation fréquentielle qui peut même être bien supérieure au quart de ton si on le désire.

Cette différence de comportement peut s'expliquer par le fait que le système $A$ translate les deux fréquences de résonance $F$ et $F$ de la même quantité quelquesoit la valeur de $h$, la mise en oeuvre de $A$ cónserve done 1'harmonicité $F / 2 F$. Le système $B$ translate la fréquence de résonance $F$ sans translater ${ }^{2} F_{2}:{ }^{1}$ la mise en oeuvre de $B$ ne conserve pas l'harmonicite $F_{2} / 2 F_{1}$.

Nous avons là une vérification expérimentale de l'idée énoncée par Bouasse et Benade (paragraphe 1) sur le cas particulier d'un système à 2 pics d'impédance d'importances équivalentes.

\section{3-3 Influence de l'embouchure sur la facilité d'émission}

En analysant précisément le spectre de pression interne au bec (figure 3) d'une "note qui roule", il apparait que les fréquences du spectre peuvent être interprétées comme combinaison linéaire de deux fréquences $F_{1}$ et $F_{2}$ inharmoniques (régime bipériodique). 


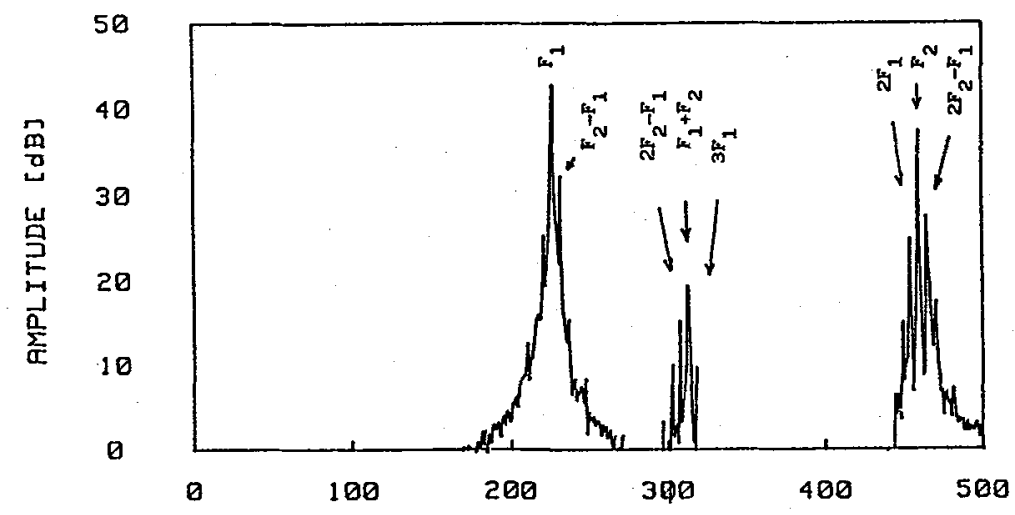

FREQUENCE [Hz]

Figure 3 : Spectre de pression interne obtenu pour le doigté de sol grave avec le tube $B$ en dérivation de hauteur $h=60 \mathrm{~mm}$. Ce spectre d'amplitude est obtenu par FFT de 1024 points sur une fenetre rectangulaire de 1.024 s. $F_{1}=225.8 \mathrm{~Hz}$ et $F_{2}=460.6 \mathrm{~Hz}$.

Nous avons vérifié que $F$ et $F$ ne sont pas les deux premières fréquences de résonance du résonateưr mals du résonateur complété à 1 'entrée par le volume équivalent à l'anche.

L'impédance d'anche $\mathrm{Za}$ caractérisée par une correction de longueur $\Delta l$ à l'entrée du résonateur, est critique pour la valeur de $F / 2 F$. Nous avons pu vérifier que "relacher l'embouchure" (augmenter $\Delta \ell^{2}$ peút compenser l'inharmonicité introduite par le système $B$, ce qui donne ainsi à l'instrumentiste un moyen de stabiliser l'oscillation.

\section{4- Conclusion :}

Lors de la mise au point du saxophone quart de ton nous avons précisé l'interprétation des courbes d'impédance d'entrée en prenant en considération une première approximation de l'embouchure de l'instrumentiste. Nous avons pu préciser le lien entre l'harmonicité des fréquences de résonance et la facilité d'émission sur le cas particulier du saxophone alto. Ces expériences ont été réalisées à la fois par des saxophonistes et avec une "bouche artificielle".

\section{Références :}

11 Bouasse H., Instruments à vent Vol. 1 p. 115. Paris, réédition Blanchard (1986).

12/ Benade A.H., Fundamentals of Musical Acoustics, Oxford U.P., New-York (1976).

/3/ Gilbert J., Etude des instruments de musique à anche simple : extension de la méthode d'équilibrage harmonique, rôle de l'inharmonicité des résonances, mesure des grandeurs d'entrée. Thèse, Université du Maine, Le Mans (1991).

14/ Nederveen C.J., Acoustical Aspects of Woodwind Instruments. Frits Knuf, Amsterdam (1969). 